\title{
Machine Vision of Clustering Identical Parts in Cellular Manufacturing
}

\author{
Prabhu $\mathbf{M}^{1 *}$ and Ramesh Kumar $\mathbf{K}^{2}$ \\ ${ }^{1}$ Research Scholar, Karpagam University, Coimbatore, Tamil Nadu, India \\ ${ }^{2}$ Principal, Asian College of Engineering and Technology, Coimbatore, Tamil Nadu, India
}

*Corresponding author: Prabhu M, Research Scholar, Karpagam University, Coimbatore, Tamil Nadu, India, Tel: + 91-422-64711; E mail: prabhubemech@yahoo.co.in

Received date: November 17, 2014; Accepted date: February 25, 2015; Published date: February 28, 2015

Copyright: () 2015 Prabhu M, et al. This is an open-access article distributed under the terms of the Creative Commons Attribution License, which permits unrestricted use, distribution, and reproduction in any medium, provided the original author and source are credited.

\begin{abstract}
Business process Re-Engineering (BPR) involves the Radical Redesigning complex and dynamic processes in Research and development (R\&D) organizations with multi-layer projects is a difficult task. Previous researchers have proposed many perceptive approaches for BPR utilizing intuition and subjective judgment from "experts" at various stages of their implementation. The proposed method facilitates the reorganization of R\&D processes to automate assembly lines; we have tested and evaluated various methods on part discrimination for a model conveyer line. An advanced method is developed on tracking and picking up a specified part among a variety of parts on a moving conveyer. The method consists of a part shape discrimination analysis following the image processing of CCD camera shots for on-conveyer parts retrieving stored images from a collection by comparing features automatically extracted from the images themselves. The common features used are mathematical measures of colour, texture or shape; hence virtually all current CBIR systems.
\end{abstract}

Keywords: Cellular manufacturing; Group technology; Business process re-engineering

\section{Introduction}

GT is a methodology for organizing work into independent groups each responsible for the production of a given family of products [1]. GT simply states that similar things should be done similarly. These problems are also known as the machine-part cell formation (CF) problems where parts and machines in a manufacturing process are assigned to independent cells so that the machine utilization within a cell is maximized and the between-cell movement of parts is minimized [2-5]. We extend the machine-part CF method to model the system process to automatically track and pick specific parts from a moving conveyor. As initial problem to identify and group parts in $R \& D$ organizations. Our model is simple, easily implemented, and it is based on well-known and widely used GT concepts. This paper is, we present brief review to the criteria for evaluation of clustering solution of identical parts are picked and grouped into cells using the proposed CBR techniques. The important step in the design of cellular manufacturing system is to identify manufacturing the part family and machine groups, consequently to form cells. The scope of this article entitled "Machine Vision of Clustering Identical Parts in Cellular Manufacturing System" aims at find in accurate results using clustering rather than comparing our files provides an efficient approach to cluster and visualize the multimedia by constructing schematic field with the set of multimedia tags. The schematic field is circle region with several gravitational sources (tags) to locate the multimedia by calculating the positions in field.

\section{Literature Review}

One of the oldest-established means of accessing pictorial data is retrieval by its position within an image. Accessing data by spatial location is an essential aspect of geographical information systems (section 5.6.8), and efficient methods to achieve this have been around for many years (e.g. Chock et al. (1984), Roussopoulos et al. (1988). Similar techniques have been applied to image collections, allowing users to search for images containing objects in defined spatial relationships with each other. Improved algorithms for spatial retrieval are still being proposed. Spatial indexing is seldom useful on its own, though it has proved effective in combination with other cues such as colour, Smith and Chang (1997a) and shape. Another method giving interesting results is retrieval by appearance. Two versions of this method have been developed, one for whole-image matching and one for matching selected parts of an image. The part-image technique involves filtering the image with Gaussian derivatives at multiple scales, and then computing differential invariants; the whole-image technique uses distributions of local curvature and phase. In the next section, we present the details of our model and show that our model is (1) simple to understand and use; (2) automated and does not require human expert intervention; (3) easily implementable with minimum computing power, and (4) based on well-known and widely used GT concepts [6-13].

\section{Proposed System}

A part shape discrimination analysis following the image processing of CCD camera shots for on-conveyer parts retrieving stored images from a collection by comparing features automatically extracted from the images themselves. An efficient approach to cluster and visualize the image of parts of a machine by constructing semantic field with a set of multimedia tags. A semantic field is a circle region with several gravitation sources (tags) to locate a multimedia by calculating the positions in the field. Multimedia classification is an important form to multimedia retrieval. Clustering method is introduced which uses gravitation based semantic field to cluster and visualize the multimedia resources. Multimedia resources can be displayed in a visual circle region and the similar multimedia resources can be recommended according to the distances in the semantic field. When gravitation sources are assigned with different weights, the semantic association distance or similarity degree will also change. According the 
Page 2 of 4

adjustment of weights of gravitation sources, the multimedia resources will also change.

\section{Visual information retrieval}

We design table to accommodate images use traditional text columns to describe the schematic significance of the images for example the picture automobile or its engine six or eight cylinders and use information retrieval type for the image to permit content base queries based on intrinsic attribute of the image how closely its colour and shape match a picture of a specific automobile. Specific composite data type used that combines visual information retrieval and appropriate text numeric and date attributes. The primary advantage is to reduce time and effort to require image base informations. The frequent adding and updating of images massive data base it's not practical to require manual entry for all the attributes that might be needed for queries and content base retrieval provides increased flexibility and practical value it's also useful in providing ability to query on attributes such as texture or structure that are difficult to represent key words. Data base application where CBR is useful (i.e) where the queries schematic of the form, "find object that look like this one"-include (Figure 1).

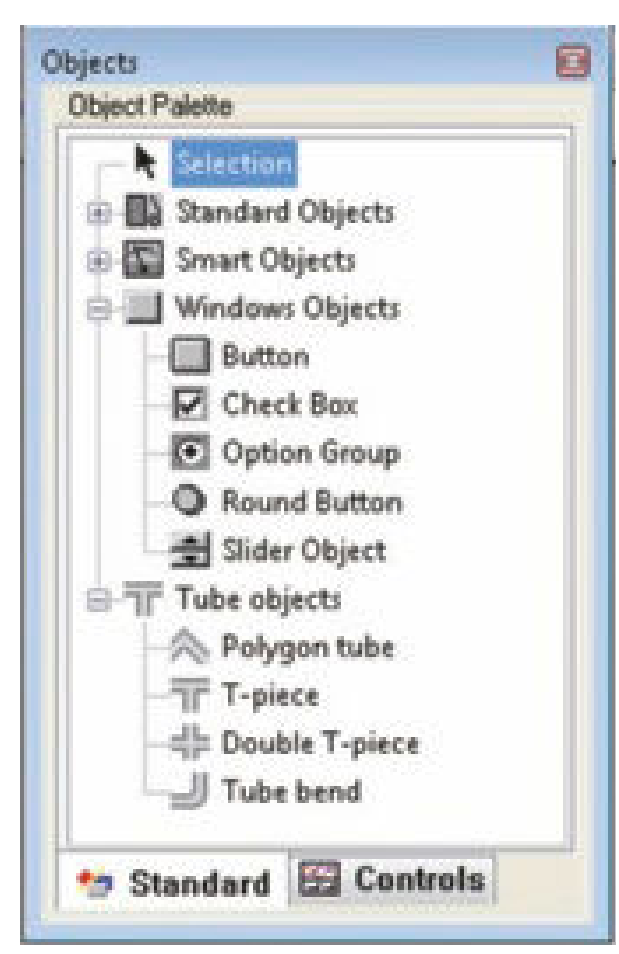

Figure 1: Object Palettes of the Graphics Designer.

\section{Working of CBR}

A Content based retrieval system processes the information contained in image data and creates an abstraction of its content in terms of visual attributes. CBR every image inserted into database to analyze and compact representation of its content stored in a feature vector or signature with following visual attributes such as global colour, local colour, texture, structure, shape.

\section{Matching operations}

When we match images, we assign an importance measure or weight to each of the visual attributes and visual information retrieval calculate a similarity measure for each visual attribute.

$$
\begin{aligned}
& \operatorname{vecSim}(\mathrm{M} 1, \mathrm{M} 2)=\frac{\sum_{i=1}^{n} \omega_{1 i} \times \omega_{2 i}}{\sqrt{\sum_{i=1}^{n} \omega_{1 i}{ }^{2} \sum_{i=1}^{n} \omega_{2 i}{ }^{2}}} \ldots . . \text { Eq. (1) } \\
& \operatorname{jacSim(M1,M2)}=\frac{\left|M_{1} \cap M_{2}\right|}{\left|M_{1} \cup M_{2}\right|} \ldots \ldots . . \text { Eq. (2) }
\end{aligned}
$$

Weight: Each weight value reflects how sensitive the matching process should be to the degree of similarity and dissimilarity between two images. In our paper weights are calculated by finding similarity between two tags.

Score: The similarity measure for each visual attribute is calculated as the score or distance between two images with respect to that attribute.

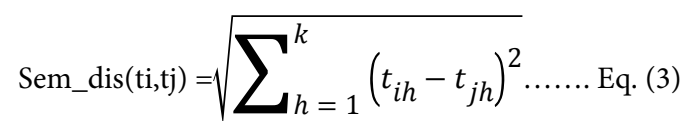

Manhattan distance can be also used to calculate the distances between vectors.

$$
M a n_{-} \operatorname{dis}(t i, t j)=\sum_{h=1}^{k}\left(t_{i h}-t_{j h}\right)^{2}
$$

Similarity calculations: In reality when images are matched the degree of similarity depends on a weighted sum reflecting the weight and distance of all four of the visual attributes of the comparison image and the test image.

Threshold value: When we match images we assign a threshold value. If the weighted sum of the distances for the visual attributes is less than or equal to the threshold, the images match if the weighted sum is greater than the threshold the images do not match.

The number of matches returned generally increases as the threshold increases. Setting the threshold to 100 would return all images as matches. Such a result of course, defeats the purpose of content base retrieval.

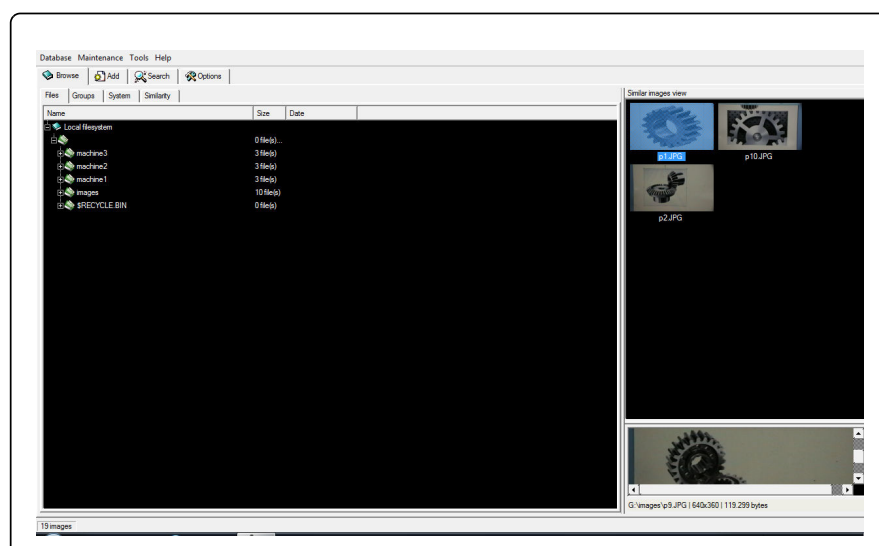

Figure 2: Spin image representation-a. 


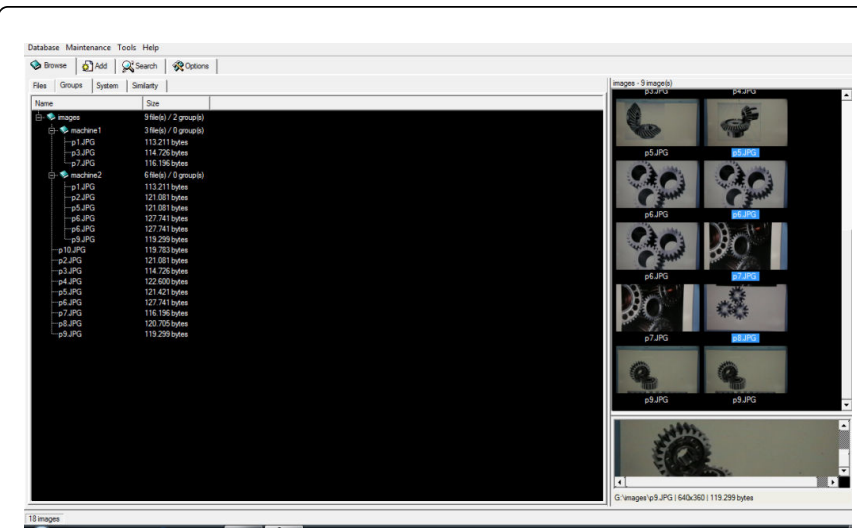

Figure 3: Spin image representation-b

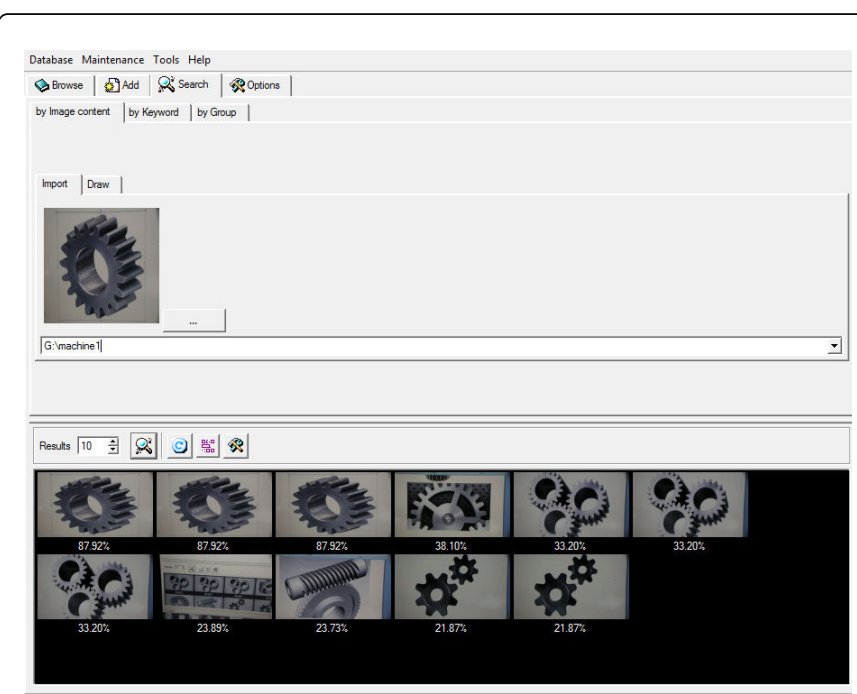

Figure 4: Spin image representation-c

\section{Criteria for Evaluation of Clustering Solutions}

Several authors have discussed the criteria for good clustering in data modeling. Moody and Flitman proposed a set of nine principles and associated measurable criteria for clustering. Our algorithm is designed to meet the following seven criteria mainly drawn from the data modeling and GT literature:

- Semantically meaningful: Parts familiar with the task domain should find the clusters logical and coherent;

- Completeness: Decomposition should cover all of the parts and machines and no parts or parts to machine should be left out.

- Non-redundancy: Each parts and machine should be in one, and only one, cluster;

- Fully connected: All the parts in a cluster should be connected to each other, via job paths that are within the cluster;

- Maximal cohesion within clusters: To the extent possible, all parts within a cluster should be closely related to each other;

- Minimal coupling between clusters: To the extent possible, parts in different clusters should not be closely related to each other; and
- High degree of modularity: Provided all of the other criteria are satisfied, a solution with a greater number of clusters is preferred to a solution with smaller number of clusters.

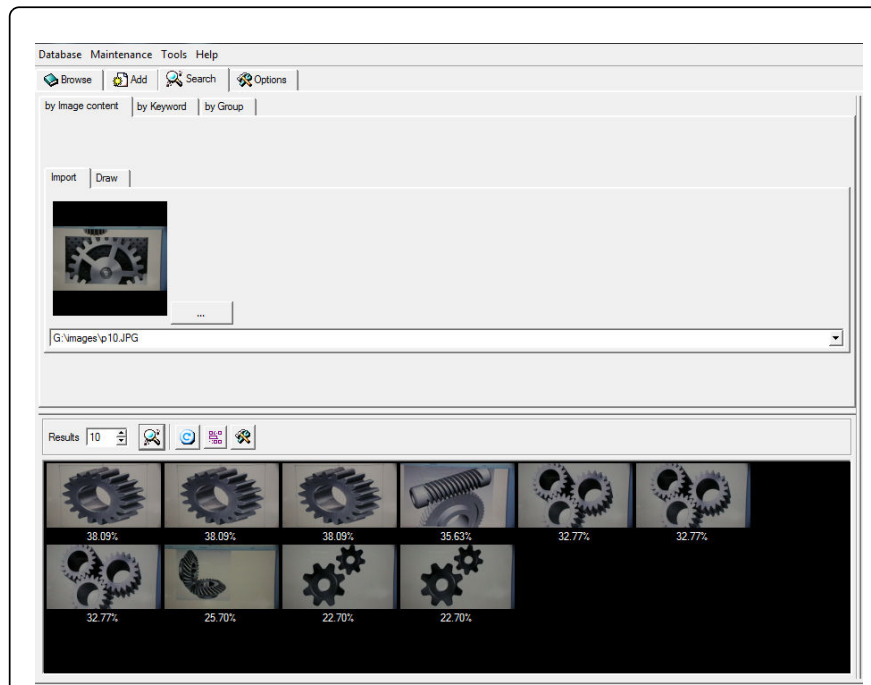

Figure 5: Spin image representation-d

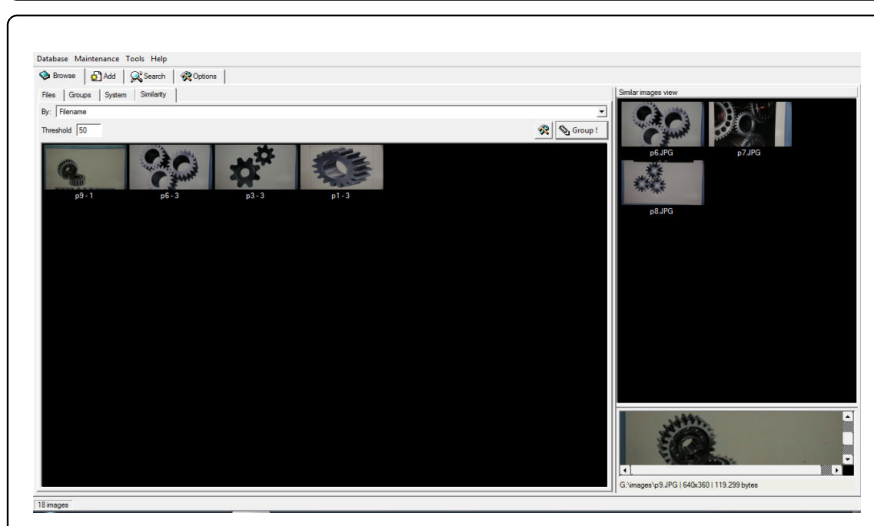

Figure 6: Spin image representation-e

\section{Conclusions}

Description vectors have been clustered using fuzzy clustering so as to take the centers of the clusters as signatures of the spin image representation as shown in the Figures 2 to 6 . The optimal number of clusters is derived considering two functions that express a measure of under- and over-partitioning, respectively. The optimal number of clusters is the number that minimizes the sum of the two functions representing the trade-off between under- and over-partitioning. Finally, similarity between spin image signatures of $3 \mathrm{D}$ objects is obtained considering the permutation that minimizes the sum of distances between the corresponding cluster centers. Some tags are not added, such new tags can be added by the user as per their requirements. Content based retrieval can be done to improve quick search and match to real time objects further study to collect and assemble part to machine separate cells to avoid human intervention for identify similar parts and group into cell we had design a software using multimedia, CCD camera to capture and compare identical 
Citation: Prabhu M, Ramesh Kumar K (2015) Machine Vision of Clustering Identical Parts in Cellular Manufacturing. Ind Eng Manage 4: 154. doi:10.4172/2169-0316.1000154

Page 4 of 4

similar grouping of parts on a conveyor line into separate cell. Future work to group parts to machine cell formation using multimedia and also to identify exceptions of parts.

\section{References}

1. Adenso DB, Lozano S, Eguira I (2005) Part-machine grouping using weighted similarity coefficients. Computers \& Industrial Engineering 48: 553-570.

2. Adil GK, Rajamani D, Strong D (1993) A mathematical model for cell formation considering investment and operational cost. European Journal of Operational Research 69: 330-341.

3. Albadawi Z, Bashir HA, Chen M (2005) A mathematical approach for the formation of manufacturing cell. Computers \& Industrial Engineering 48: 3-21.

4. Arvindh B, Irani SA (1991) Principal components analysis for evaluating the feasibility of cellular manufacturing without initial-part matrix clustering. International Journal of Production Research 32: 1909-1938.

5. Burgess AG, Morgan I, Vollmann TE (1993) Cellular manufacturing: its impact on the total factory. International Journal of Production Research 31: 2059-2077.
6. Boctor FF (1991) A linear formulation of the machine-part cell formation problem. International Journal of Production Research 29: 343-356.

7. Reisman A, Kumar A, Motwani J, Cheng CH (1997) Cellular manufacturing: A statistical review of the literature. Operations Research 45: 508-520.

8. Mitrofanov SP (1966) Scientific Principles of Group Technology, Part I. National Lending Library of Science and Technology, Boston, MA.

9. Opitz H, Eversheim W, Wienhal HP (1969) Work-piece classification and its industrial applications. International Journal of Machine Tool Design and Research 9: 39-50.

10. Agarwal A, Sarkis J (1998) A review and analysis of comparative performance studies on functional and cellular manufacturing layouts. Computers and Industrial Engineering 34: 77-89.

11. Van Z, Kazai G, Lalmas M (2006) INEX2005 multimedia track, Advances in XML Information Retrieval Lecture Notes in Computer Science. Springer.

12. Smeulders AWM, Worring M, Santini S, Gupta A, Jain R (2000) Contentbased image retrieval at the end of the early years. Pattern Analysis and Machine Intelligence, IEEE Transactions on 22:1349-1360.

13. Jain AK, Hong L, Pankanti S, Bolle R (1997) An identity authentication system using fingerprints, Proceedings of the IEEE 65:1365-1366. 